\title{
Tunable Electrical Properties of Aligned Single-Walled Carbon Nanotube Network-based Devices: Metallization and Chemical Sensor Applications
}

\author{
Young Lae Kim**† and Myung Gwan Hahm** \\ *Department of Mechanical and Industrial Engineering, Northeastern University, Massachusetts 02115, USA \\ **Department of Materials Science and Engineering, Inha University, Incheon 22212, Korea
}

(Received September 27, 2017; Revised October 19, 2017; Accepted October 19, 2017)

\begin{abstract}
Here we report the tunable electrical properties and chemical sensor of single-walled carbon nanotubes (SWCNTs) networkbased devices with a functionalization technique. Formation of highly aligned SWCNT structures is made on $\mathrm{SiO}_{2} / \mathrm{Si}$ substrates using a template-based fluidic assembly process. We present a Platinum (Pt)-nanocluster decoration technique that reduces the resistivity of SWCNT network-based devices. This indicates the conversion of the semiconducting SWCNTs into metallic ones. In addition, we present the Hydrogen Sulfide $\left(\mathrm{H}_{2} \mathrm{~S}\right)$ gas detection by a redox reaction based on SWCNT networks functionalized with 2,2,6,6-Tetramethylpiperidine-1-oxyl (TEMPO) as a catalyst. We summarize current changes of devices resulting from the redox reactions in the presence of $\mathrm{H}_{2} \mathrm{~S}$. The semiconducting (s)-SWCNT device functionalized with TEMPO shows high gas response of $420 \%$ at $60 \%$ humidity level compared to $140 \%$ gas response without TEMPO functionalization, which is about 3 times higher than bare s-SWCNT sensor at the same $\mathrm{RH}$. These results reflect promising perspectives for real-time monitoring of $\mathrm{H}_{2} \mathrm{~S}$ gases with high gas response and low power consumption.
\end{abstract}

Key words : Carbon nanotube, Resistivity, Metallization, Hydrogen sulfide, Sensor

\section{Introduction}

$\mathrm{C}$ arbon nanotubes (CNTs) have been considered as a possible replacement for copper electrical interconnects for future electronics with their outstanding failure current densities $\left(>10^{9} \mathrm{~A} \cdot \mathrm{cm}^{-2}\right){ }^{1)}$ However, since naturally grown SWCNTs contain a mixture of metallic and semiconducting nanotubes, there is a need to develop a process that can convert semiconducting nanotubes into metallic ones within the structures. Semiconducting nanotubes have too high resistivities to utilize for interconnects. Therefore, a singlestep and simple method is highly desirable, which can simultaneously convert semiconducting SWCNTs into metallic ones. It also possibly increases the conductance of metallic SWCNTs. It is essential to address these technological challenges before SWCNT network devices can be realistically implemented as futuristic electronics. We have also shown that the conductance of individual multi-wall carbon nanotubes (MWCNTs) can be improved significantly by decorating its surface with Pt nanoclusters. Field theoretical analysis confirm that this is caused by the increase in the number of conductance channels of the nanotubes. Ab-initio density functional theory (DFT) calculations also show that Pt-nanoclusters can convert semiconducting SWCNTs into metallic ones, and improve the conductance of metallic

${ }^{\dagger}$ Corresponding author: Young Lae Kim

E-mail : kim.youn@husky.neu.edu

Tel : +1-971-334-9982 Fax : +1-617-373-2921 nanotubes as well. ${ }^{2)}$ Upon Pt decoration, the electrical resistivity of these SWCNT network-based structures decreased by $45 \%$, with a $52 \%$ drop on the average for the narrower channels.

Recently, moreover, there has been significant interest in using SWCNT-based nanomaterials as chemical sensors due to several benefits of SWCNT structures such as light weight, high electrical conductivity and large surface area, and outstanding sensing performance. Particularly, CNTs are a growing demand due to their high electron mobility and large current capability, which can potentially help in reducing power consumption of the sensor and increasing temperature stability and chemical inertness of CNTs. It may provide a stable and robust platform to detect specific gas. ${ }^{3,4)} \mathrm{H}_{2} \mathrm{~S}$ is a harmful gas which can cause lung damage and asphyxiation when exposed to it. ${ }^{5,6)}$ Thus, the monitoring of $\mathrm{H}_{2} \mathrm{~S}$ is essential for safety issue because this gas is widely found in industry such as natural gas and petroleum, and is a by-product in the manufacture of synthetic rubber, dyes and the tanning of leather. ${ }^{7}$ Although there are several $\mathrm{H}_{2} \mathrm{~S}$ gas sensors available commercially, their sensing monitors include high power consumption with high operating temperatures, interference with other gases, and short lifetime and high cost. ${ }^{8)}$ Here, we present molecular functionalization of 2,2,6,6-Tetramethylpiperidine-1oxyl (TEMPO) as a catalyst on the surface of SWCNTs and its efficient detection of $\mathrm{H}_{2} \mathrm{~S}$ gas by a redox reaction at room temperature. 


\section{Experimental Procedure}

\subsection{Metallization of SWCNT networks}

As-received SWCNTs in the DI-water based solution are a mixture containing approximately $2 / 3^{\text {rd }}$ semiconducting nanotubes and $1 / 3^{\text {rd }}$ metallic carbon nanotubes. In aligned architectures, due to the high resistance of the semiconducting nanotubes (about 2 - 3 orders of magnitude larger than metallic nanotubes), ${ }^{9)}$ only $1 / 3^{\text {rd }}$ metallic CNTs actively conduct current, and the rest remain dormant for semiconducting CNTs. This degrades the potential performance of SWCNT network-based device. Therefore, it is critical to change the electrical property of semiconducting nanotubes into metallic ones for the interconnect applications. Moreover, we found that narrower SWCNT devices tend to have higher resistivity, due to the reduced number of metallic conduction paths within the SWCNT networks. Kar et al. has shown that Pt-decoration of carbon nanotubes can be an effective method for increasing the number of conduction channels near the Fermi level, which increases the conductance of multi-wall carbon nanotubes. ${ }^{10)}$ Based on this study, Pt nanoclusters were electrochemically decorated on

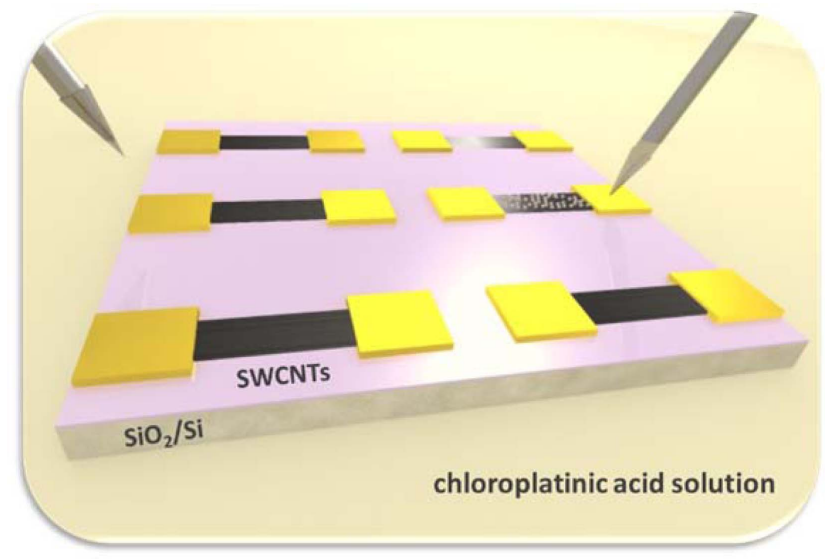

Fig. 1. Schematic for Pt-decoration of SWCNT networkbased devices in a dilute chloroplatinic acid solution. A negative electric potential on one of the electrodes (directly contacted, right needle) induces (indirect) negative potentials on the neighboring devices due to the capacitive effect of the oxide-coated a silicon wafer. the SWCNT networks without disturbing their aligned structures. Fig. 1 is a schematic of the $\mathrm{Pt}$ decoration process on SWCNTs. To decorate Pt nanoparticles on the surface of SWCNT networks, we immersed the assembled SWCNT devices in a $5 \mathrm{mM}$ chloroplatinic acid solution. When a negative potential is applied to the electrodes of the device in the solution, Pt ions having positive charges are selectively nucleated on the surface of the electrodes and the SWCNT networks.

\subsection{Functionalization of SWCNT networks}

Figure 2(a) shows the experiment setup for $\mathrm{H}_{2} \mathrm{~S}$ gas detection. SWCNT network device was assembled by a templateguided fluidic assembly process and then electrodes were fabricated on top of aligned SWCNT networks. This process enabled us to build SWCNT network devices at predetermined locations and with uniform density of SWCNT devices for a large-scale fabrication. For the safety issue of $\mathrm{H}_{2} \mathrm{~S}$ gas test and precise controlling of detection, we set up the SWCNT-based sensor devices with wire bonding to reduce the electrical noise from ambient environments and vibrations. Fig. 2(b and c) shows optical and SEM images with a wire bonding process in the overall structure and individual test structure, respectively.

\section{Results and Discussion}

SWCNT network-based devices were evenly decorated with smaller Pt nanoparticles $(<5 \mathrm{~nm})$ on the surface of carbon nanotubes. Fig. 3(a) inset is an SEM image of SWCNT arrays with Pt nanoclusters on the surface of structures. SEM image and energy dispersive x-ray spectroscopy (EDS) maps of an SWCNT array with Pt nanoparticles were shown in Fig. 3(a). EDS map for Pt shows very small amounts of $\mathrm{Pt}$ decoration. The EDS data along with the spectral analysis is confirmed with the fact that a few nanometers of Pt-clusters were uniformly decorated over the surface of aligned SWCNT network device. The resistivity value of SWCNT networks with different widths (before and after Pt-decoration) is shown in Fig. 3(b). In order to calculate the resistivity, the length and width (at 5 positions along the length and width) of the devices were obtained

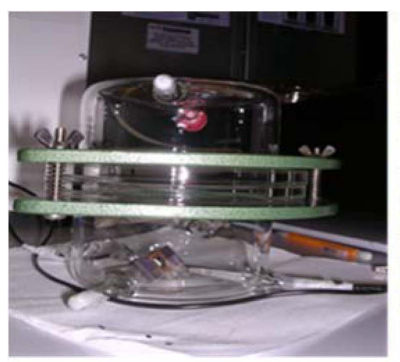

(a)

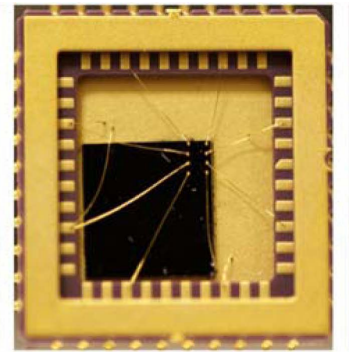

(b)

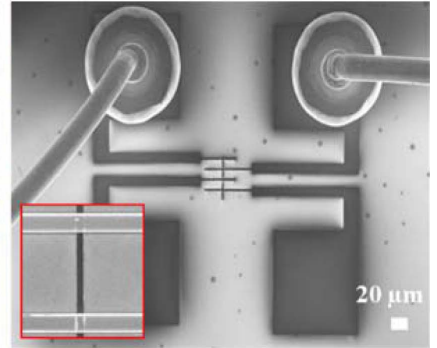

(c)

Fig. 2. $\mathrm{H}_{2} \mathrm{~S}$ gas sensing environment based on SWCNT networks. (a) Glass chamber setup for controlled environment measurements, (b) optical image of SWCNT devices in the wired bonded chip, and (c) SEM image of sensing area of SWCNT networks with electrodes and wire bonders. 


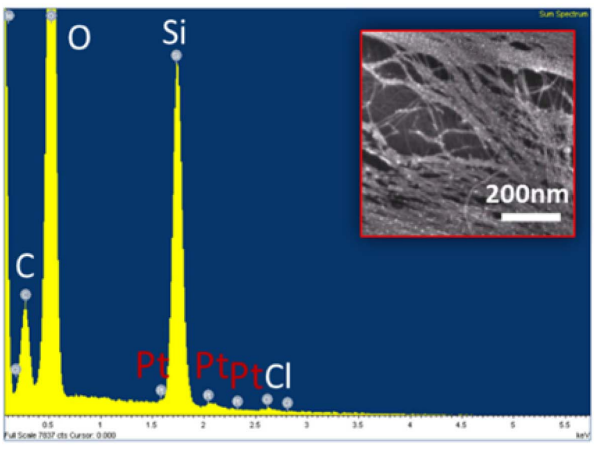

(a)

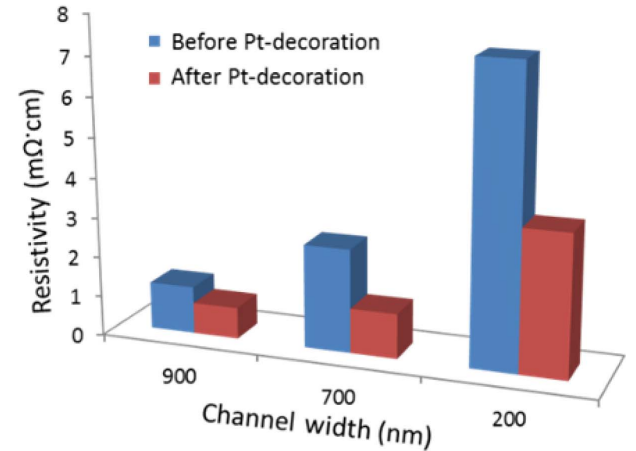

(b)

Fig. 3. (a) Typical SEM image of Pt-decoration on the surface of SWCNT networks and its corresponding energy dispersive x-ray spectroscopy (EDS) map of Pt decoration, showing that Pt nanoparticles were decorated on the SWCNT networks. (b) Resistivity of the devices with different channel widths before and after Pt decoration.

from SEM images for widths and from AFM measurement for their heights. Data show that as the device widths of the SWCNT networks decrease, the values of resistivity increase, indicating a dominant semiconducting SWCNTs in the narrower widths. Hence, the average decrease of resistivity after Pt-decoration is different from different channel widths. In the case of a $200 \mathrm{~nm}$ channel width, resistivity was reduced by $51 \%$ changing from $7.4 \mathrm{~m} \Omega \cdot \mathrm{cm}$ to 3.6 $\mathrm{m} \Omega \cdot \mathrm{cm}$. The changes to drop in resistivity were found to be about $46 \%$ and $33 \%$ for $500 \mathrm{~nm}$ and $700 \mathrm{~nm}$ channel networks after the Pt-decoration respectively. For the larger percentage drop in resistivity for the $200 \mathrm{~nm}$ channel width, the fraction of semiconducting nanotubes in the SWCNT networks was larger compared to larger channel widths, which causes a higher percentage change.

To extend the concept of metallization process of SWCNT networks, we study on the detection of $\mathrm{H}_{2} \mathrm{~S}$ gas on functionalized SWCNT networks with TEMPO. Fig. 4(a) depicts a schematic on the interaction of $\mathrm{H}_{2} \mathrm{~S}$ molecules on SWCNTs functionalized with TEMPO molecules. SWCNT networks serve as an active channel layer with high surface area to the chemical interaction with $\mathrm{H}_{2} \mathrm{~S}$ gas. Since TEMPO composes of stable nitroxyl group provided by the adjacent four methyl groups, it has been widely used as a radical trap and as a reagent in organic synthesis. ${ }^{11,12)}$ Thus it is also able to oxidize the gaseous $\mathrm{H}_{2} \mathrm{~S}$ so that it can be utilized as a sensor molecule for a chemical sensor to detect $\mathrm{H}_{2} \mathrm{~S}$ molecules. For each concentration of $\mathrm{H}_{2} \mathrm{~S}$, we evaluated gas response $\mathrm{S}$ defined by $S=\left\{\left(I_{i}-I_{\text {gas }}\right) / I_{i}\right\} \times 100$, where $I_{i}$ is the initial current and $\mathrm{I}_{\text {gas }}$ is the changed current after injection of $\mathrm{H}_{2} \mathrm{~S}$ gas. Fig. 4(b) shows gas response of a bare s-SWCNT device and a device functionalized with TEMPO as a function of relative humidity $(\mathrm{RH})$. The gas response of bare s-SWCNT devices was increased significantly to $150 \%$ at $60 \%$ at $\mathrm{RH}$. $\mathrm{H}_{2} \mathrm{O}$ molecules can be adsorbed on the surface of SWCNTs and act as electron donors in a p-type semiconductor reducing the hole density in s-SWCNTs resulting in the current increase. ${ }^{13)}$ This indicates that moisture is one of important factors in gas response of the sensor and practical applications. To maximize gas response based on the effect of water vapors, TEMPO was used as a homogeneous catalyst for redox reaction of $\mathrm{H}_{2} \mathrm{~S}$ and $\mathrm{H}_{2} \mathrm{O}$. As shown in Fig. 4(b), sSWCNT devices functionalized with TEMPO showed $420 \%$ gas response at $60 \% \mathrm{RH}$ compared to $140 \%$ gas response without TEMPO functionalization, which is about 3 times higher than bare s-SWCNT sensor at the same RH. To understand the sensing mechanism of increasing response to $\mathrm{H}_{2} \mathrm{~S}$ gas on CNTs, we focus on the redox reactions of various molecules which exist near active CNT channels and TEMPO with humidity condition. These CNT channels

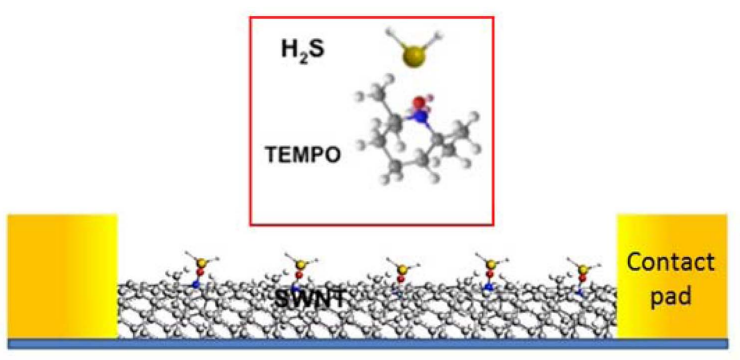

(a)

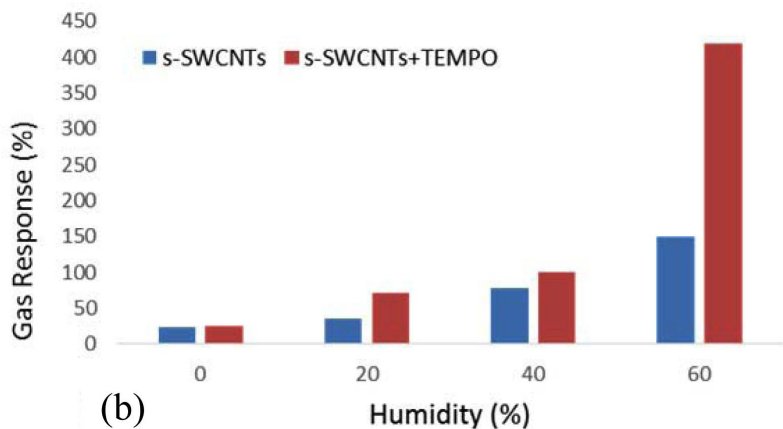

(b)
Humidity (\%)

Fig. 4. (a) Schematic on the detection of $\mathrm{H}_{2} \mathrm{~S}$ molecules on SWCNTs functionalized with TEMPO molecules. (b) Gas response of $\mathrm{H}_{2} \mathrm{~S}$ gas sensor depending on humidity for s-SWCNTs and s-SWCNTs+TEMPO. 100 ppm concentration of $\mathrm{H}_{2} \mathrm{~S}$ is used. 
show the p-type characteristics in the ambient condition. ${ }^{14)}$ This is attributed to the fact that the CNT is p-doped due to the oxygen adsorption, through which each $\mathrm{O}_{2}$ molecule donates a hole to CNTs or takes an electron away from the CNTs. These adsorbed $\mathrm{O}_{2}$ molecules existing in the form of $\mathrm{O}_{2}^{-}$participate in our proposed redox reaction by donating electrons back to the CNTs resulting in less p-doped CNT channels under humidity condition. In the presence of TEMPO, TEMPO is oxidized to be TEMPO+ with powerful positive, which is a significant product for $\mathrm{H}_{2} \mathrm{~S}$ dissociation, while $\mathrm{H}_{2} \mathrm{O}$ molecules with $\mathrm{O}_{2}$ molecules from $\mathrm{O}_{2}^{-}$are reduced. These redox reactions between $\mathrm{H}_{2} \mathrm{~S}$ gas and CNTs with TEMPO functionalization could explain the increase of gas responses.

\section{Conclusions}

In conclusion, we have fabricated highly aligned SWCNT network-based devices using a template-guided fluidic assembly process for the tunable electrical properties of their structures and the $\mathrm{H}_{2} \mathrm{~S}$ gas sensor with TEMPO. We have demonstrated a Pt-decoration technique to enhance the overall electrical conductivities of these structures. Ptdecoration leads to significantly reduce the electrical resistivity depending on its channel width, with evidence of overall conversion of the semiconducting nanotubes into metallic ones in some cases. This result is in agreement with the calculations of band gap and Landauer conductances. The processes are relatively simple to implement and easily scalable to wafer levels, which involved in the fabrication and performance enhancement via Pt-decoration. In addition, we present s-SWCNT network-based sensor with functionalized with TEMPO can detect selectively $\mathrm{H}_{2} \mathrm{~S}$ gas up to $420 \%$ gas response at $60 \% \mathrm{RH}$, which has 3 times higher gas response than bare s-SWCNT sensor at the same RH. This SWCNTbased sensor with TEMPO can open new possibilities in development of multi-functional chemical gas senor that can effectively detect multiple gaseous species. This offers promising perspectives of SWCNT-TEMPO network devices for real-time monitoring of $\mathrm{H}_{2} \mathrm{~S}$ gases with outstanding gas response and lower power consumption. Such a small size of the device will also demonstrate futuristic integration of sensors with low power electronics.

\section{REFERENCES}

1. B. Wei, R. Vajtai, and P. Ajayan, "Reliability and Current Carrying Capacity of Carbon Nanotubes," Appl. Phys. Lett., 79 [8] 1172 (2001).
2. S. Kar, C. Soldano, L. Chen, S. Talapatra, R. Vajtai, S. Nayak, and P. Ajayan, "Lüttinger Liquid to Al'tshulerAronov Transition in Disordered, Many-Channel Carbon Nanotubes," ACS Nano, 3 [1] 207-12 (2009).

3. M. S. Strano, C. A. Dyke, M. L. Usrey, P. W. Barone, M. J. Allen, H. Shan, C. Kittrell, R. H. Hauge, J. M. Tour, and R. E. Smalley, "Electronic Structure Control of SingleWalled Carbon Nanotube Functionalization," Science, 301 [5639] 1519-22 (2003).

4. E. Snow, F. Perkins, E. Houser, S. Badescu, and T. Reinecke, "Chemical Detection with a Single-Walled Carbon Nanotube Capacitor," Science, 307 [5717] 1942-45 (2005).

5. H. Peng, Y. Cheng, C. Dai, A. L. King, B. L. Predmore, D. J. Lefer, and B. Wang, "A Fluorescent Probe for Fast and Quantitative Detection of Hydrogen Sulfide in Blood," Angew. Chem., Int. Ed., 50 [41] 9672-75 (2011).

6. K. Abe and H. Kimura, "The Possible Role of Hydrogen Sulfide as an Endogenous Neuromodulator," J. Neurosci., 16 [3] 1066-71 (1996).

7. B. Yang, S. Wang, S. Tian, and L. Liu, "Determination of Hydrogen Sulfide in Gasoline by Au Nanoclusters Modified Glassy Carbon Electrode," Electrochem. Commun., 11 [6] 1230-33 (2009).

8. A. Chowdhuri, V. Gupta, K. Sreenivas, R. Kumar, S. Mozumdar, and P. Patanjali, "Response Speed of SnObased HS Gas Sensors with CuO Nanoparticles," Appl. Phys. Lett., 841180 (2004).

9. A. Vijayaraghavan, K. Kanzaki, S. Suzuki, Y. Kobayashi, H. Inokawa, Y. Ono, S. Kar, and P. Ajayan, "Metal- Semiconductor Transition in Single-Walled Carbon Nanotubes Induced by Low-Energy Electron Irradiation," Nano Lett., 5 [8] 1575-79 (2005).

10. S. Kar, C. Soldano, L. Chen, S. Talapatra, R. Vajtai, S. Nayak, and P. Ajayan, "Lüttinger Liquid to Al'tshulerAronov Transition in Disordered, Many-Channel Carbon Nanotubes," ACS Nano, 3 [1] 207-12 (2009).

11. V. Jeena and R. S. Robinson, "Convenient Photooxidation of Alcohols Using Dye Sensitised Zinc Oxide in Combination with Silver Nitrate and TEMPO," Chem. Commun., 48 [2] 299-301 (2012).

12. T. Saito, Y. Nishiyama, J. L. Putaux, M. Vignon, and A. Isogai, "Homogeneous Suspensions of Individualized Microfibrils from TEMPO-Catalyzed Oxidation of Native Cellulose," Biomacromolecules, 7 [6] 1687-91 (2006).

13. A. Zahab, L. Spina, P. Poncharal, and C. Marliere, "Water-Vapor Effect on the Electrical Conductivity of a Single-Walled Carbon Nanotube Mat," Phys. Rev. B, 62 [15] 10000 (2000)

14. P. G. Collins, K. Bradley, M. Ishigami, and A. Zettl, "Extreme Oxygen Sensitivity of Electronic Properties of Carbon Nanotubes," Science, 287 [5459] 1801-4 (2000). 